\title{
Traduire
}

Revue française de la traduction

$218 \mid 2008$

De traduction en retraduction

\section{Continuer à traduire la voix de Cortázar}

\section{Sylvie Protin}

\section{(2) OpenEdition \\ Journals}

Édition électronique

URL : http://journals.openedition.org/traduire/899

DOI : 10.4000/traduire.899

ISSN : 2272-9992

Éditeur

Société française des traducteurs

Édition imprimée

Date de publication : 15 juin 2008

Pagination : 65-76

ISSN : 0395-773X

\section{Référence électronique}

Sylvie Protin, «Continuer à traduire la voix de Cortázar », Traduire [En ligne], 218 | 2008, mis en ligne le

15 juin 2008, consulté le 04 mai 2019. URL : http://journals.openedition.org/traduire/899 ; DOI

$10.4000 /$ traduire.899 


\title{
Continuer à traduire la voix de Cortázar*
}

\section{Sylvie Protin}

\author{
Pour ne citer qu'un exemple,
} si son discours ce soir-là a été greffé sur le son a, dont il est capable d'extraire d'interminables modulations, changements harmoniques et dérivations vers le e ou le o (mettons, toute la gamme des aae, aea, aee, aoa, aoo, aeoa, aeeoo, etc.), il suffira que je me trouve dans l'impossibilité d'établir le pont logique entre deux états de la matière du discours pour que tout l'édifice s'écroule. La rage de mon pince-gueule ne connaît alors plus de bornes et j’en ai ressenti souvent les conséquences. Julio Cortázar, "Les discours du pince-gueule ".

Parler, ou écrire, ce n'est pas seulement produire un énoncé, c'est aussi se dire soi-même disant le monde : c'est là qu'émerge la voix. En parlant, on dresse - sans toujours le vouloir - un portrait de soi au monde. En tant qu'individu d'abord : on dit sa conscience particulière du monde, l'ordre qu'on lui prête et la place qu'on s'y accorde. Mais on dresse aussi un portrait de soi en tant que membre d'une collectivité : le groupe social, la profession, la classe d'âge, etc. parlent aussi par notre bouche ou sous notre plume.

Cette question de la voix touche donc tous les actes de parole et, partant, tous les aspects de la traduction, qu'il s'agisse d'interprétation, de traduction audiovisuelle, de traduction pragmatique ou littéraire. Toutefois, il est évident que la littérature constitue depuis toujours un terrain privilégié où l'écriture tente sciemment de faire naître une ou des voix ; il n'est qu'à mentionner le théâtre. La voix comme véhicule de l'identité et de la conscience individuelle face à l'ordre social est même devenue absolument centrale dans toute la littérature du XXe siècle - qu'on repense par exemple à l'oralité chez

* Cette communication a été présentée par l'auteur à l'occasion de la $6 \mathrm{e}$ Journée de la traduction professionnelle (Lyon, 4 avril 2008), ndlr. 
Louis-Ferdinand Céline. C'est dans cette lignée que se place l'œuvre de l'écrivain argentin Julio Cortázar.

Qui est Cortázar ? Tout d'abord, il s'agit d'un auteur mort, en 1984. Pourquoi commencer par là ? C'est que cela définit le point de vue du traducteur de 2008(1). Il se trouve face à une œuvre mondialement reconnue, une œuvre achevée et donc produite dans un temps désormais révolu. Or si l'on continue à traduire un auteur plus de vingt ans après sa mort, c'est que quelque chose de sa voix a pris, qu'elle résonne encore dans les sensibilités contemporaines. Traduire un auteur mort, c'est continuer à tisser des liens vivants avec une voix d'outre-tombe.

Cortázar est donc né en 1914 et mort en 1984. C’est un véritable maître du fantastique et de l'humour. Il a développé une littérature dont les singularités sont presque sensuelles : ses textes éduquent le regard, enseignent à voir jaillir du quotidien la coïncidence incroyable, l'étrange, l'insolite comme une manifestation poétique ou fantastique. Cortázar, c'est aussi une voix aisément reconnaissable.

Sa voix physique, d'abord, était tout à fait marquante : il avait la cadence lente et chaloupée de l'espagnol d'Argentine, une grande attention au rythme interne de la phrase, qu'il dramatisait volontiers, et enfin, marque indélébile, un défaut de prononciation en espagnol l'empêchait de rouler les « $\mathrm{R}$ ", qu'il grasseyait. Sa voix de papier est, elle aussi, clairement identifiable : pour savoir si le texte qu'il venait d'écrire était bon, Cortázar le lisait à haute voix en s'enregistrant sur un magnétophone, de façon à savoir s'il avait le "swing ". Il nommait ainsi, par référence au jazz, une sorte de tempo, d'entraînement tout musical de la phrase littéraire(2).

(1) Ces lignes sont écrites à l'occasion de la parution du volume Quarto consacré à Julio Cortázar (Nouvelles, histoires et autres contes, Gallimard, mars 2008, 1416 pages). L'auteur du présent article a dirigé ce volume, dans lequel sont reprises les traductions déjà existantes de Laure Guille-Bataillon, Karine Berriot, Françoise Campo-Timal et Isabelle Dessomes ; elle a également traduit les textes inédits qui y sont rassemblés.

(2) Pour entendre l'auteur lire certains de ses textes, on pourra par exemple se reporter au site http://www.juliocortazar.com.ar/suvoz.htm. Même si l'on ne parle pas espagnol, on sera sensible au rythme de cette écriture très orale. 
En réalité, la voix a deux fonctions chez Cortázar : d'une part, elle peut marquer la présence d'une subjectivité de narration, voire franchement la présence de l'auteur qui cherche à entretenir ainsi une relation directe avec le lecteur. D'autre part, elle peut servir à incarner un personnage d'une façon très économique, en faisant abstraction de toute description ou mise en contexte.

Ce trait distinctif de l'écriture de Cortázar a été très bien senti par celle qui fut pour lui une grande amie et sa principale traductrice en Français : Laure Guille-Bataillon. En dialoguant avec l'auteur, elle a inventé dans notre langue une voix de Cortázar basée sur la musicalité, le tempo et les jeux de langage(3). Elle a ainsi créé un timbre qui est à son tour devenu clairement identifiable pour les lecteurs français, à tel point qu'il existe désormais une attente face aux nouvelles traductions. Un lecteur a ainsi dit un jour à propos d'un texte nouvellement traduit : "Je reconnais mon Cortázar». Quelque chose de l'ordre de la voix était à ses yeux passé, non pas de l'espagnol à notre traduction, mais de son archétype français de Cortázar à notre texte.

Cet archétype est aussi intéressant que périlleux car se trouver face à une œuvre globale, c'est aussi avoir à traduire les marges de l'œuvre : les textes plus rares, plus difficiles, les œuvres de jeunesse, la correspondance, etc. Ainsi, il nous a été donné de traduire l'œuvre critique de Cortázar, constituée de textes écrits entre les années 1940 et les années 1980. La voix bien identifiable de cet auteur est bel et bien là dès les années 50, mais il en va tout autrement pour les premiers textes. En effet, sur le plan du style, notre lecteur ne pourrait pas dire "Je reconnais mon Cortázar» dans la production des années 40. Les textes de jeunesse sont très charpentés logiquement, les propositions s'y imbriquent les unes dans les autres dans un jeu de subordonnées souvent subtil. La syntaxe y est complexe, très intellectuelle ; elle réclame une grande attention au lecteur pour suivre parfois certaines phrases sur plusieurs pages, au cours de nombreuses concessions ou incises qui

(3) Rappelons ici que Cortázar, lui-même traducteur et réviseur, relisait toutes les traductions de ses textes vers le français et dialoguait volontiers avec ses traducteurs. 
nuancent le propos et le font avancer. Il existe clairement un mouvement dans ces textes, mais c'est le rythme tenu d'une pensée qui progresse par balancements logiques et non un rythme sensuel ou musical(4). Les textes postérieurs obéissent à une tout autre syntaxe, fondée non plus sur la subordination mais sur la parataxe et la juxtaposition. Les relations entre les propositions ne sont plus logiques mais analogiques. Cette fois-ci, c'est le rythme de l'oralité qui guide l'écriture; dans un texte comme "Felisberto Hernández: lettre en main propre", on entend clairement la voix archétypique de Cortázar(5).

Il y a donc eu un Cortázar d'avant Cortázar ; il est partiellement identifiable au pseudonyme de Julio Denis utilisé par l'auteur jusqu'au début des années 40. Traduire la voix de tête de Julio Denis, c'est comprendre le chemin parcouru pour que naisse la voix de bouche de Julio Cortázar. Entre ces deux syntaxes, il y a deux visions du monde :

(4) Voir par exemple cet incipit de "L'Urne grecque dans la poésie de John Keats" (traduction inédite ; texte original de 1946, repris dans Obra crítica 2) : "C'est par deux voies que semble s'opérer l'accès du monde moderne aux ordres spirituels de l'antiquité gréco-latine, toutes les fois qu'un désir de connaissance et d'identification profonde l'a poussé à se retourner vers lui, en quête d'une prise de contact qui puisse lui redonner des valeurs oubliées parfois au cours de l'évolution historique de l'Europe. Par ces deux voies - qui tendent à s'exclure l'une l'autre -, l'esprit moderne a voulu revenir aux inspirations esthétiques du classicisme et faire corps avec ces forces créatrices et leurs expressions artistiques, afin ensuite de les recréer. La première voie (déjà incomparablement ouverte par la Renaissance et sa redécouverte du monde classique) connaît ses périodes les plus fleuries dans le classicisme français du XVII siècle et dans les formes analogues, quoique particulières, de ce même mouvement dans l'Angleterre et l'Allemagne du XVIII. . "

(5) Voir par exemple l'incipit (traduction parue dans La Nouvelle Revue Française $\mathrm{n}^{\circ}$ 570, juin 2004 ; texte original de 1983, repris dans Obra crítica 3): "Felisberto, tu sais (je n'écrirai pas " tu savais ", nous avons toujours aimé, toi et moi, enfreindre les temps des verbes comme une juste revanche sur l'autre temps, ces montres et ces calendriers qui nous éreintent), tu sais que les prologues des œuvres complètes ou des anthologies sont presque toujours amidonnés comme des cours magistraux, et ça, ça ne nous plaît pas du tout, à nous qui préférons lire des nouvelles ou raconter des histoires ou marcher dans la ville entre deux petits verres de vin." " 
la première repose sur l'appréhension d'un univers complexe, hiérarchisé, fait de subordination et où le sujet semble peiner à exister ; la seconde fait état d'une vision du monde plus immanente, moins médiatisée par l'intellect : le rapport aux choses et aux autres se veut direct, transgressant volontiers la grammaire et la logique pour atteindre un plus grand contact.

De ceci découlent deux conclusions et une question. Première conclusion : l'oralité en littérature n'a rien d'une plate copie de la parole orale. C'est au contraire une déconstruction du beau style qui vise à construire une autre esthétique, ce processus passant par une modification profonde de la syntaxe (comme Picasso dégradait un dessin tout réaliste pour fabriquer le trait cubiste). Deuxième conclusion : la syntaxe et la ponctuation elles aussi se traduisent. Les ressorts de la voix et de l'oralité dépassent largement les choix possibles en matière de lexique ; par-delà le niveau de langue, c'est la danse de la conscience avec le monde, son rythme, qui donne la sensation de la voix.

Quant à la question, ce serait : qu'est-il arrivé à cet auteur pour qu'il modifie ainsi son style ? Pendant ces années de transition d'une voix à une autre, Cortázar a été traducteur, aussi bien technique que littéraire. Il a notamment traduit Gide, Giono, Cocteau, Yourcenar ou encore Poe, autrement dit, il a travaillé sa palette en reconstituant en espagnol ces écritures si diverses. Il semble ainsi que la traduction lui ait servi de laboratoire pour atteindre cette adéquation de la langue aux mouvements de la conscience, processus qu'il nommait « la pourriture de mon ancien style ». Montrer en français le chemin parcouru depuis la voix de tête des textes de jeunesse jusqu'à l'élaboration de cette voix de bouche exige de longs étirements linguistiques, une patiente gymnastique de la syntaxe. Cela permet aussi, en tant que critique cette fois, de mieux comprendre le parcours de ce grand écrivain. La traduction reste ainsi un espace privilégié où se produit une lecture toute particulière des textes, une lecture avec l'oreille.

Mais, disions-nous, la voix a également une autre fonction dans les fictions cortazariennes : celle de faire émerger l'épaisseur des personnages 
en faisant l'économie complète de leur description. En effet, la façon dont les personnages parlent dénote leur tempérament, leur milieu social, leurs sentiments, leur relation avec l'interlocuteur, etc. Or, le plus souvent, cet effet est obtenu par l'infraction aux codes de la grammaire et de la convenance. Cette pratique pousse le traducteur dans ses retranchements et l'engage à jouer au même jeu de transgression jubilatoire dans sa langue d'arrivée. Nous proposons ci-après trois situations problématiques posées par la traduction de cette voix narrative chez Cortázar.

Un premier exemple de personnage entièrement défini par sa voix serait cette poule mutante(6) :

Por escrito gallina una

Con lo que pasa es nosotras exaltante. Rápidamente del posesionadas mundo estamos hurra. En un inofensivo aparentemente cohete lanzado Cañaveral americanos Cabo por los desde. Razones se desconocidas por órbita de la desvió, y probablemente algo al rozar invisible la tierra devolvió a. Cresta nos cayó en la paf, y mutación golpe entramos de. Rápidamente la multiplicar aprendiendo de tabla estamos, dotadas muy literatura para la somos de historia, química menos un poco, desastre ahora hasta deportes, no importa pero : de será gallinas cosmos el, carajo qué.
Par écrit poule une

Ce qui arrive nous exaltant est. Nous rapidement appartenu le monde a hourra. Il y a eu une fusée inoffensive apparemment lancée Cap Américains Canaveral par les depuis. Raisons pour des inconnues elle a l'orbite de dévié, et probablement lâché a quelque chose en frôlant d'invisible la terre. Ça crête nous est tombé sur la paf, et commencé à muter nous avons. Nous vite les de multiplications avons appris tables, nous fortes très littérature sommes pour l'histoire de la, chimie pour la un peu moins, nulles jusqu’à présent les pour sports, dommage mais : le aux poules cosmos appartiendra, dieu de nom.

(6) Le texte original, intitulé "Sociedad anónima ", est tiré de La vuelta al día en ochenta mundos (Siglo XXI editores, 1998, p. 170) ; la traduction est parue dans Nouvelles, histoires et autres contes, op. cit., p. 624. 
Ce texte est très efficace : la mutation a doté les poules de la parole, mais elles ne sont pas fortes très pour l'ordre des propositions. Comment traduire un texte fondé sur le désordre ? Il faut d'abord remarquer que ce désordre n'est que fonctionnel ou apparent: Cortázar prend bien soin de conserver une lisibilité basée sur l'inférence. Ainsi, le début des trois premières phrases espagnoles pourrait être lu normalement. Mais il crée aussi des effets comiques, comme "Cresta nos cayó en la paf" ("Ça crête nous est tombé sur la paf »), qui sont de véritables raccourcis visuels. Pour traduire la littérarité de ce texte, c'est-à-dire le jeu avec le lecteur, le traducteur ne peut que reconstruire le même jeu, avec les mêmes contraintes : faux désordre basé sur une lisibilité relative et effets comiques de carambolage. L'ordre des propositions dans la traduction n'est donc évidemment pas le même qu'en espagnol.

Voici, dans "Société anonyme »(7), un autre personnage tout en voix : c'est le bonimenteur de foire que nous avons tous entendu sur les marchés. Son discours en espagnol est émaillé d'expressions toutes faites, de ruptures de syntaxe, d'apostrophes, d'argot et d'allusions paillardes, si bien qu'on entend réellement le personnage en train de faire l'article. Mais le traducteur, souvent, hésite un peu devant l'anacoluthe ou le juron de charretier : l'ultracorrection guette toujours. Il est alors très utile de savoir que Cortázar, qui a vécu quelque trente ans en France, a lui-même écrit une poignée de textes directement en français $(8)$. Ils démontrent que Cortázar n’a absolument pas

(7) Texte original tiré de La vuelta al día en ochenta mundos (ibid. p. 168) ; traduction parue dans Nouvelles, histoires et autres contes, p. 623.

(8) On pourra lire par exemple "Tout ira plus ou moins bien jusqu'où ", p. 566 de Nouvelles, histoires et autres contes : "Moi, je suis pour les machines, la preuve, je me promène à la campagne avec ma $4 \mathrm{~L}$ rouge Estérel, une couleur très chic, en voilà une machine obéissante comme une fleur, première, seconde, troisième, débrayage, ah qu'il fait lourd, baissons les vitres, ah, comme il pleut, actionnons l'essuie-glace zup zap, zup zap, tiens, il fait lourd à nouveau, et puis quelle voiture docile, quelle tenue de. Paf, elle s'arrête au beau milieu du trèfle ". Ailleurs dans le même texte, un exemple de registre grossier : " tu parles de ces salopes qui chient sur les dalles". 
peur du registre grossier et qu'il utilise fréquemment l'anacoluthe pour faire suivre au texte les mouvements de conscience du narrateur : ces faits de style ont leur raison d'être littéraire et c'est donc bien cela qui est à traduire. En effet, cet auteur ne conçoit pas la littérature comme le lieu de la belle langue, mais comme celui du jeu et de l'agression face au langage amidonné. Il écrit par exemple dès 1947 dans Teoría del túnel:

Les écrivains démultiplient les possibilités de la langue, l'amènent à sa limite, cherchant toujours une expression plus immédiate, plus proche du fait en soi qu'ils sentent et veulent manifester, c'est-à-dire une expression non esthétique, non littéraire, non idiomatique. L'ÉCRIVAIN EST L'ENNEMI POTENTIEL - ET AUJOURD'HUI BIEN REEL - DE LA LANGUE. Le grammairien le sait et c'est pour cela qu'il est toujours vigilant, dénonçant les abus et les transgressions, effrayé par cette lente dislocation d'un mécanisme que lui conçoit, ordonne et fixe comme une parfaite, une infaillible machine d'énonciation.

Pour être loyale envers le texte, son auteur et le lecteur, il fallait dès lors chercher des accents similaires à la voix de Cortázar en français pour faire entendre ce personnage de bonimenteur :

Plusieurs douzaines de badauds, les sprays d'insecticide sur la table à tréteaux, le stand installé à un carrefour, une chaleur liquéfiante mais notre José, la casquette en arrière, apostrophe les badauds sans aucune amabilité, le produit que j'ai l'honneur de vendre n'a pas besoin de parlotte pour épater son monde, cet insecticide à pulvériser est à la fois infaillible et bon marché, et les deux ne font pas souvent la paire. Les insecticides vendus en droguerie, c'est tout notices et boîtes en couleur, ça vous montre des mouches et des cafards en pleine agonie dramatique, mais je vous jure quil y en a un bon paquet qui sont de vraies vitamines pour l'insecte, vous pulvérisez et la bête en est toute enthousiasmée, elle vous grimpe au mur, elle volette entre les pendeloques du lustre, bref, vous lui avez rendu service et en plus ça vous a coûté quatre-vingts balles. 
Dans certains cas enfin, comme "Effets du qu'en dira-t-on »(9), la connotation sociale des voix est déterminante pour le récit. Il s'agit ici d'un micro-conte, c'est-à-dire une forme très ramassée et économique où toute une ambiance se concentre en quelques lignes. Ici, une famille argentine visiblement rurale est à table et, soudain, le téléphone sonne alors que la maison n'en est pas équipée. L'effet fantastique marche à plein. L'improbable est arrivé et les personnages essaient contre toute logique de faire comme si de rien n'était. En quelques lignes, Cortázar met en scène et dénonce l'hypocrisie d'un ordre social artificiel et rassurant. Pour ce faire, Cortázar utilise le "voseo " typique de l'oralité argentine et nomme ses personnages "la Yolanda " ou " el Pedro ", ce qui dénote une extraction populaire. Enfin, un détail final achève de brosser ce tableau si économique : la famille mange de la polenta, ce qui, en Argentine, a longtemps été l'élément fondamental du dîner dans les très nombreuses familles d'immigrants italiens. Une première traduction de ce texte a été tentée, en suivant les habitudes standard (les prénoms ne se traduisent pas et la lettre du texte doit être respectée). "El Pedro " posait bien sûr problème car " le Pedro " ne correspondait à rien dans notre univers linguistique. Le personnage avait donc été réduit à un simple "Pedro ", ce qui effaçait les connotations d'un parler rural et populaire. Cette traduction était correcte, mais l'effet du texte n'y était pas : la suspension fantastique de la fin ne semblait pas réellement fonctionner. La relecture d'un très intéressant texte de Laure Guille-Bataillon, intitulé «Traduire Cortázar avec Cortázar ", a alors été décisive :

Julio Cortázar m'expliqua, bien avant de l'écrire, pourquoi il fallait absolument que rien n'arrêtât le lecteur dans la montée progressive d'un conte bref. Cette intensité, disait-il, c'est l'élimination de toutes les idées ou situations intermédiaires, de tous les remplissages, explications ou phrases de transition que le roman demande. Il va sans dire que le traducteur sera tenu à la même rigueur, qu'il devra se garder

(9) Texte original paru dans Territorios, Siglo XXI editores, 1992, p. 80 ; traduction parue dans Nouvelles, histoires et autres contes, p. 991. 
d'exotismes qui risqueraient de détourner le lecteur de l'émotion ascendante qui doit le gagner. Or il est facile de faire de l'exotisme, il suffit souvent d'être trop fidèle au texte. C'est ainsi que pour les besoins de la cause, dans une nouvelle (Après dîner, je crois), un térou se vit déguisé en chouette, Julio Cortázar ne supportant pas l'idée que le lecteur français risque d'être distrait par un oiseau inconnu et risque de se poser, si brièvement que ce fût, des questions sur sa forme ou son chant alors que se jouait une partie autrement fondamentale (10).

En écoutant l'écho de ce dialogue entre l'auteur et sa première traductrice, le choix a été fait de revoir le texte et d'abandonner son " exotisme" au profit de son efficacité. "El Pedro " est donc devenu "Pierrot" ou « le Pierrot». De même, la polenta finale, qui renvoie plutôt à l'Italie qu'à l'Argentine pour le lecteur français (lequel n'y voit d'ailleurs pas forcément un plat du pauvre), est devenue une soupe, notre équivalent paysan du repas du soir. La version française est donc une scène acculturée, mais qui garde ainsi son efficacité fantastique. Voici la fin de ce texte en français :

La mère continue à le regarder, tout en écoutant la sonnerie qui continue encore et encore. "De toute façon, faudra bien répondre ", dit-elle enfin et sans s'adresser à personne en particulier.

"Vas-y, toi ", ordonne le père à Yolande, l'aînée.

"Mais où?", dit la Yolande.

"Faites ce que vous dit votre père, jeune fille", dit la mère.

Yolande, indécise, se lève, enlève sa serviette.

"Et qu'est-ce que je dis ?", demande-t-elle comme pour gagner du temps.

Ils se regardent tous pendant que la sonnerie, toujours plus irritée, toujours plus urgente, retentit.

"Dis que c'est un faux numéro ", ordonne le père.

"Et reviens vite, ta soupe va refroidir", ajoute la mère comme si elle voulait ôter de l'importance à toutes ces allées et venues dans la maison.

(10) L. Bataillon : "Traduire Cortázar avec Cortázar ", in Traduire, écrire (Arcane $\left.\mathrm{n}^{\circ} 17\right)$ 1991, p. 56. 
Continuer à traduire les voix de Cortázar aujourd'hui requiert donc une très grande écoute du texte initial, de ses effets, de ses fonctionnements, de ses problématiques. Pour être loyal envers cette littérarité souvent transgressive, le traducteur doit à son tour acquérir, on l'a vu, une certaine intrépidité vis-à-vis du français, de la notion de "langue littéraire " et de ses habitudes de traduction.

Pour traduire Cortázar sans Cortázar, il faut en somme prendre garde à ne surtout pas normaliser la voix de cet ennemi bien réel de la langue.

Sylvie Protin est docteur en littérature hispano-américaine et maître de conférences à l'université Lyon 2 (département de LEA), où elle enseigne la traduction et l'interprétation. Elle a participé à la création du Master 2 Traduction Littéraire et édition critique de Lyon 2, au sein duquel elle enseigne. Egalement traductrice littéraire, en particulier de Julio Cortázar, elle a dirigé le volume Nouvelles, histoires et autres contes de Cortázar chez Quarto (Gallimard) et a traduit son ouvre critique (encore à paraître).

sylvie.protin@univ-lyon2.fr

\section{Références bibliographiques}

" Pour saluer Julio Cortázar " in "La Nouvelle Revue Française ", Gallimard, $n^{\circ}$ 570, Juin 2004.

Cortázar, Julio, La vuelta al día en ochenta mundos, Siglo XXI editores, 1998.

Cortázar, Julio, Nouvelles, histoires et autres contes, trad. de l'espagnol par Karine Berriot, Françoise Campo-Timal, Isabelle Dessommes, Laure Guille-Bataillon, Sylvie Protin et Françoise Rosset. Édition publiée sous la direction de Sylvie Protin, Collection Quarto, Gallimard, mars 2008. 
Cortázar, Julio, Obra crítica 2, textes réunis par Jaime Alazraki, Ed. Alfaguara, 1994.

Cortázar, Julio, Obra crítica 3, textes réunis par Saúl Sosnowki, Ed. Alfaguara, 1994.

Cortázar, Julio, Territorios, Siglo XXI editores, 1998.

Guille-Bataillon, Laure, " Traduire Cortázar avec Cortázar » in Traduire, écrire (Arcane $\left.{ }^{\circ} 17\right), 1991$. 